\title{
INFLUENCE OF COILING IN STRESS RELAXATION OF PRESTRESSING STEEL WIRES
}

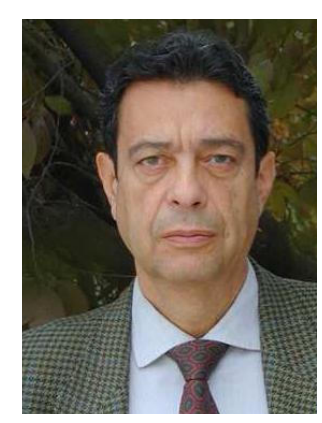

Manuel Elices

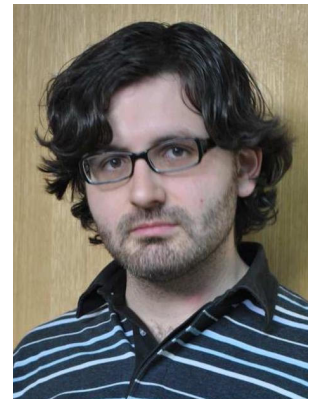

Fernando Suárez Guerra

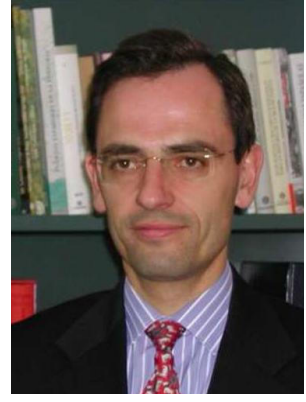

Jaime C. Gálvez

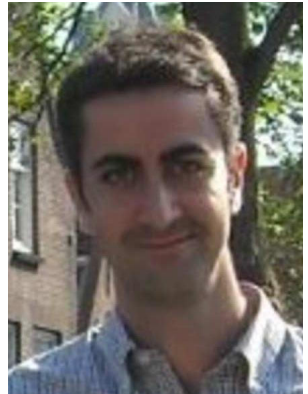

David A. Cendón

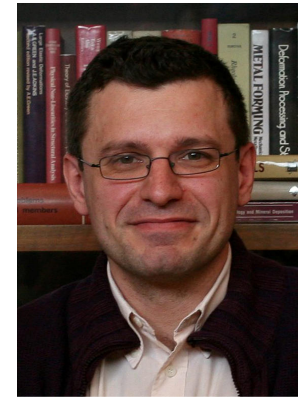

José Miguel Atienza

\begin{abstract}
The possible deleterious effects of coiling and long-time storage of coiled wires on the stress relaxation behaviour of prestressing steel wires has been checked by means of experimental work and a simple analytical model. The results show that if the requirements of Standards are fulfilled (minimum coiling diameters) these effects can be neglected. However, some other factors like previous residual stresses, long-time storage or storage at high temperatures, can trigger or emphasise this damage to the material. In the authors' opinion it is recommendable to control the final curvature of the wires after uncoiling prior to prestressing, as required in some Standards.
\end{abstract}

Keywords: Stress relaxation, Prestressing steel, Coiling diameter.

\section{Introduction}

There is experimental evidence, supported by manufacturers and constructors, that stress relaxation losses increase in coiled wires and strands, particularly after long-time storage or if coiled with small diameters.

This fact is due to the stress relaxation of the outermost stressed fibres of the wires during storage, which generates a certain plastic strain. This plastic strain is responsible for the curvature that remains when the wire is uncoiled, which evidences the appearance of additional residual stresses (as shown in Fig. 1).

There are two main factors in this process, which are limited by design codes and Standards [1]: coiling diameter and time of storage. Design codes limit the inner diameters of drums where wires and cables are coiled to avoid plastic strains. Also, some codes [2] suggest maximum values for sagitta h when measured over $1 \mathrm{~m}$ of uncoiled wire or strand (see Fig. 1). Even so, plastic strains can develop due to relaxation if enough time is allowed to pass.

\section{Experimental Programme}

\subsection{Cold-drawn wire}

For this research, wires were manufactured by cold-drawing eutectoid steel rods of $12 \mathrm{~mm}$ diameter by a commercial procedure after six drawing passes. The final diameter was $7.0 \mathrm{~mm}$. Residual stresses generated in this process where relieved by stabilising [5]. 
Standard tensile tests were performed according to ISO 15630.3 [4]. The results appear in Tab. 1. Moreover, stress relaxation tests at different initial loads, according to ASTM E328, were performed. Such results are summarised in Fig. 2.

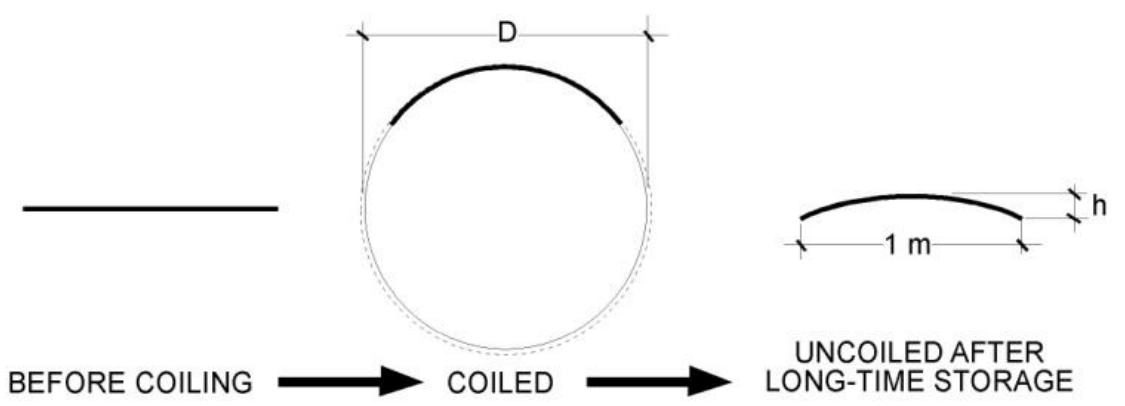

Fig.1 Sketches of wire shapes along coiling and uncoiling. The sagitta $h$ is the distance from the midpoint of the wire arch (when uncoiled) to the midpoint of its chord, when the chord is $1 \mathrm{~m}$.

Tab. 1 Tensile properties of $7 \mathrm{~mm}$ diameter steel wires

\begin{tabular}{|c|c|c|c|}
\hline$\sigma_{0.1}(\mathrm{MPa})$ & $\sigma_{0.2}(\mathrm{MPa})$ & $\sigma_{\text {uts }}(\mathrm{MPa})$ & $\varepsilon_{\mathrm{m}}(\%)$ \\
\hline 1679 & 1704 & 1823 & 5.79 \\
\hline
\end{tabular}

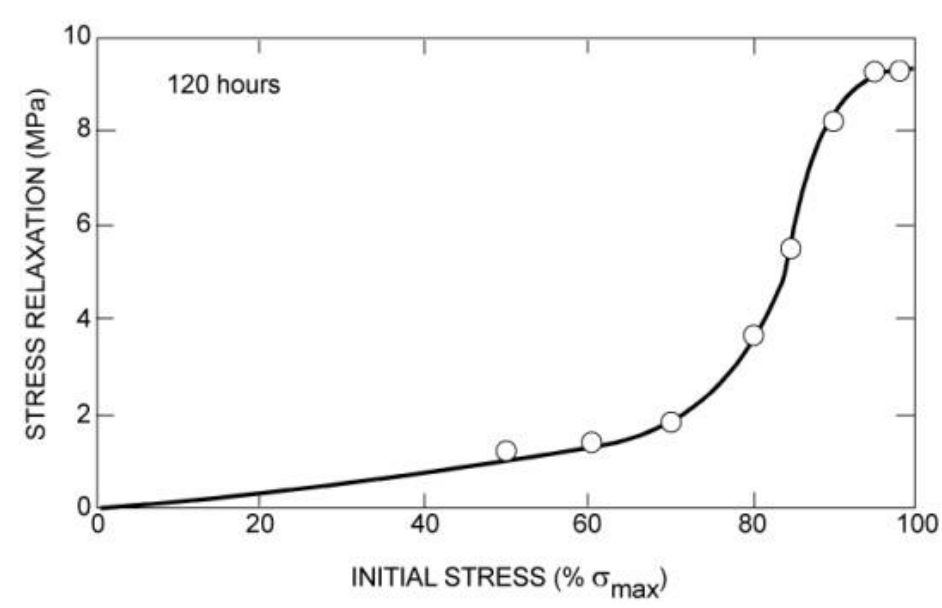

Fig.2 Stress relaxation losses, after 120 hours, for different initial loads.

\subsection{Coiling and uncoiling of wires}

Some Standards recommend a minimum value of the coiling diameter, usually 225 times the wire diameter [1]. In order to test the influence of coiling diameters, two batches of wires were coiled at lower diameters than recommended and another one at the minimum diameter recommended by the Standards. All three batches remained coiled for 120 hours.

After uncoiling, wires displayed a certain curvature, corresponding to circles of $500 \mathrm{~mm}$, $1000 \mathrm{~mm}$, and $9000 \mathrm{~mm}$ diameter, respectively.

\subsection{Stress relaxation tests}

Relaxation tests were performed at two different initial loads -70 and $80 \%$ of the ultimate tensile load - at room temperature and for up to 120 hours. Results are shown in Tab. 2.

Tab. 2 Relaxation losses at 120 hours (measured and predicted) after uncoiling. Also, data of average surface residual stresses after uncoiling and sagitta.

\begin{tabular}{|l|l|l|l|l|}
\hline Uncoiled diameter & Measured Relaxation & Predicted Relaxation & Residual & $\mathrm{h}(\mathrm{mm})$ \\
\hline
\end{tabular}




\begin{tabular}{|c|cc|cc|c|c|}
\hline$(\mathrm{mm})$ & \multicolumn{2}{|c|}{ losses (\%) } & \multicolumn{2}{c|}{ losses $(\%)$} & stresses* & \\
& $70 \%$ uts & $80 \%$ uts & $70 \%$ uts & $80 \%$ uts & $(\mathrm{MPa})$ & \\
\hline initial straight & 1.8 & 3.7 & - & - & - & 0 \\
9000 & 1.9 & 4.6 & 2.3 & 4.4 & 80 & 28 \\
1000 & 4.0 & 6.7 & 4.2 & 6.7 & 100 & 500 \\
500 & 5.5 & 6.4 & 5.9 & 7.8 & 120 & - \\
\hline
\end{tabular}

* Average surface residual stresses.

\section{Relaxation losses prediction}

A simple analytical model was developed in order to predict the influence of coiling diameter and storage time in the stress relaxation of steel wires.

This model allows to keep track of the behaviour of steel fibres through the whole process that affects the wire: coiling, long-time storage, uncoiling and stress relaxation test. This process is sketched in Fig. 3 and can be tracked as follows:

- Profile 0.- Initial state: No stresses.

- Profile 1.- After coiling, a bending moment is applied

- Profile 2.- After some time in coil: Tensile stresses relax according to Fig. 2.

- Profile 3.- After uncoiling: The stress profile is obtained by subtracting the above mentioned external moment to balance the actual loads.

- Profile 4.- At the beginning of the relaxation test at 0.70 uts. Some fibres are more loaded than 0.70 uts. and, hence, higher stress losses should be expected. Also, some fibres are less loaded than 0.70 uts but, as Fig. 2 shows, increases are more critical than decreases.

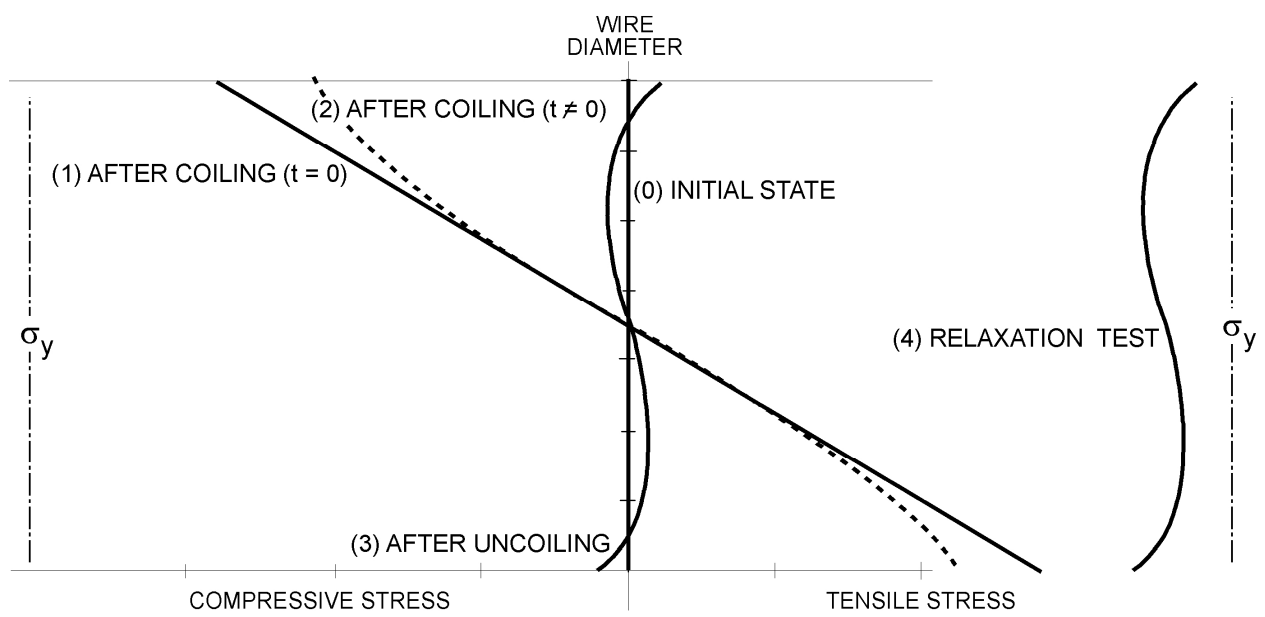

Fig.3 Stress profiles during coiling, storage, uncoiling and initial relaxation test.

No initial residual stresses.

\subsection{Comparison with experimental results}

Input data to perform computations were the stress-strain curve of the steel wire and the relaxation curve for different initial stresses recorded at 120 hours, as shown in Fig.2. The agreement is satisfactory (Tab. 2), considering the simplicity of the model. 


\section{Conclusions}

1. The experience of practitioners that coiling at small diameters and after a long-time can increase stress relaxation losses, has been experimentally confirmed.

2. A very simple model is able to predict and explain the evolution of stress relaxation after uncoiling.

3. If the requirements of Standards are fulfilled (minimum coiling diameters), the effects of coiling can be neglected. However, some other factors like previous residual stresses, long-time storage or storage at high temperatures, can emphasise this damage to the material. In the authors' opinion it is recommendable to control the final curvature of the wires after uncoiling prior to prestressing, as required in some Standards.

The authors gratefully acknowledge the financial support for the research provided by the Spanish Ministerio de Ciencia e Innovación under grants BIA-2008-03523 and MFOM-01/07. F. Suárez also gratefully acknowledges the grant supplied by Fundación Agustín de Betancourt. Authors also express their gratitude to CICYT for financial support through Project CONSOLIDER-INGENIO 2010 and to Mr. José Miguel Martínez Palacio, for his invaluable aid in the plotting of the figures included in this paper.

\section{References}

[1] EN 10138:2009. Prestressing steels. CEN, Brussels. Sections 10.3.1 and 10.3.2.

[2] EHE-08. Instrucción de Hormigón Estructural. Section 70.2.1.1.

[3] Ruíz Hervías J., Luzin V., Prask H. Gnaeupel-Herold T. and Elices M. (2006) Effect of thermomechanical treatments on residual stresses measured by neutron diffraction in colddrawn steel rods. Mater Sci Eng A, 435-436, 725-735.

[4] ISO 15630. Steel for the reinforcement and prestressing of concrete. Test methods. Part 3: Prestressing steel. CEN, Brussels.

[5] Atienza J.M. and Elices M. (2007) Role of residual stresses in stress relaxation of prestressed concrete wires. J Mater Civil Eng ASCE, 8, 703-708.

\section{Prof. Manuel Elices, CSc., C.Eng.}

E.T.S. de Ingenieros de Caminos Departamento de Ciencia de Materiales c/ Profesor Aranguren s/n 28040 Madrid, Spain

政 $\quad+34915433974$ +34915437845

;) melices@mater.upm.es

\author{
Fernando Suárez Guerra, C.Eng. \\ E.T.S. de Ingenieros de Caminos \\ Departamento Ingeniería Civil: \\ Construcción \\ c/ Profesor Aranguren s/n \\ 28040 Madrid, Spain \\ 漁 $\quad+34913366723$ \\ (;) fsuarez@caminos.upm.es
}

